Case Report

\title{
Aggressive nasopalatine duct cyst with nasal involvement
}

\author{
Pandya D. ${ }^{1}$, Dey S. ${ }^{2}$, Bhattacharya M. $^{3}$, Singh P. ${ }^{4}$ \\ ${ }^{1}$ Dr. Divya Pandya, Assistant Professor, Department of Oral Medicine and Maxillofacial Radiology, ${ }^{2}$ Dr. Soumadip Dey, \\ Assistant Professor, Department of Oral and Maxillofacial Surgery, ${ }^{3}$ Dr. Maumita Bhattacharya, Assistant Professor, \\ Department of Oral Pathology and Microbiology and Forensic Odontology, ${ }^{4}$ Dr. Pooja Singh, Assistant Professor, \\ Department of Pedodontic and Preventive Dentistry, all authors are affiliated with Kusum Devi Sundar Lal Dugar Jain \\ Dental College and Hospital, Kolkata, India.
}

Corresponding Author: Dr. Divya Pandya, Assistant Professor, Department of Oral Medicine and Radiology, Kusum Devi Sundar Lal Dugar Jain Dental College and Hospital, Kolkata, India. Dr. Divya Pandya, L 30/1 Bose Para Road, Kamdahari, Garia, Kolkata, West Bengal, India, E-mail: divyapandya854@gmail.com

\begin{abstract}
Nasopalatine duct cyst is a non-odontogenic developmental cyst typically located in the maxillary midline between the tooth roots of central incisors, these cysts are infrequent and can often be misdiagnosed as periapical lesion or cyst. In this article, we present a case of which was clinically and radio graphically provisionally diagnosed as nasopalatine duct cyst in a 62 year old male patient with complaint of swelling in midline of palate. The lesion was surgically removed, and histo pathologically confirmed the provisional diagnosis, thus concluding that it can be a diagnostic dilemma in clinical and radiological examinations.
\end{abstract}

Keywords: Cysts, Developmental, Naso palatine, Non-odontogenic

\section{Introduction}

Nasopalatine duct cyst (NPDC) is the most common non-odontogenic, developmental cyst of non-neoplastic nature. Its location is peculiar and specific in that it affects the midline anterior maxilla. Thenasopalatine duct communicates the nasal cavity with the anterior region of the upper maxilla. During fetal development the duct gradually narrows until one or two central clefts are finally formed on the midline of the upper maxilla. The nasopalatine neurovascular bundle is located within the duct and emerges from its intra-bony trajectory through the nasopalatine foramen $[1,2]$.

NPDC was first described by Meyer in 1914, who wrongly identified it as a paranasal sinus. In the past, NPDC, was termed as anterior palatine cyst or incisive canal cyst arising from embryologic remnants of nasopalatine duct and was regarded as fissural cysts formed between the two maxillae. World Health Organization in 1998 classified these lesions as nonodontogenic developmental cyst along with nasoalveolar and naso-labial cyst. Although they are most common non-odontogenic jaw cysts, they accountfor only $1 \%$ of all maxillary cysts $[1,3]$.

NPDC is commonly seen in $4^{\text {th }}$ and $5^{\text {th }}$ decade of life with slight male predilection. Clinically it may be asymptomatic and get discovered during routine radiographic examination. Larger lesion presents as a swelling over anterior maxilla, located between the tooth roots of maxillary central incisors. As the presentation of NPDC mimics common periapical lesion like radicular cyst, it is not uncommon to see evidence of endodontic treatment of associated teeth due to previous misdiagnosis of the pathology[1,3-6].

\section{Case Report}

A 62 year old male patient reported with the chief complaint of a swelling in upper front tooth region of jaw since 2 months. Swelling was insidious in onset and gradually increased to present size with no pain or discharge. Patient revealed a history of trauma few years back in same region. There was no relevant extraoral finding or lymphadenopathy. The patient's medical history was non-contributory.

\footnotetext{
Manuscript received: $2^{\text {nd }}$ March 2018

Reviewed: $10^{\text {th }}$ March 2018

Author Corrected: $17^{\text {th }}$ March 2018

Accepted for Publication: $21^{\text {st }}$ March 2018
} 


\section{Case Report}

Intraoral examination revealed a well-defined round to oval solitary nodular swelling in mid-line hard palate in nasopalatine region just beyond palatine rugae, involving both sides of mid-palatine raphe, measuring approximately 1.5 $\mathrm{cm} \times 1.5 \mathrm{~cm}$ extending posteriorly to the mesial aspect of maxillary $1^{\text {st }}$ premolars. On palpation, swelling was soft in consistency, non tender, compressible, non-reducible and non-fluctuant (Figure 1).

Pulp vitality testing showed vital 11 and 21. Intraoral periapical radiograph of maxillary anterior region revealed a diffuse radiolucency between roots of central incisors, which was not too appreciable, so a Cone Beam Computed Tomographic (CBCT) image of maxilla was recommended for 3-dimensional evaluation of lesion.

Axial, sagittal and coronal sections with 3-dimensional reformatted images were obtained and assessed to make the following report - CBCT revealed a well-defined unilocularhypodense lesion in anterior maxilla in 11, 21 regions.

The lesion extended from incisive canal opening to the floor of nasal fossa superinferiorly and from labial to palatal cortical plate labiopalatally from 13 to 23 region, causing expansion, thinning and destruction of labial cortical plate with perforation of floor of nasal fossa.

The lesion measured $1.5 \mathrm{~cm}$ mesiodistally and supero-inferiorly and $1.4 \mathrm{~cm}$ laterally. The lesion was bordered by very thin irregular sclerotic margin except few areas (Figure 2).

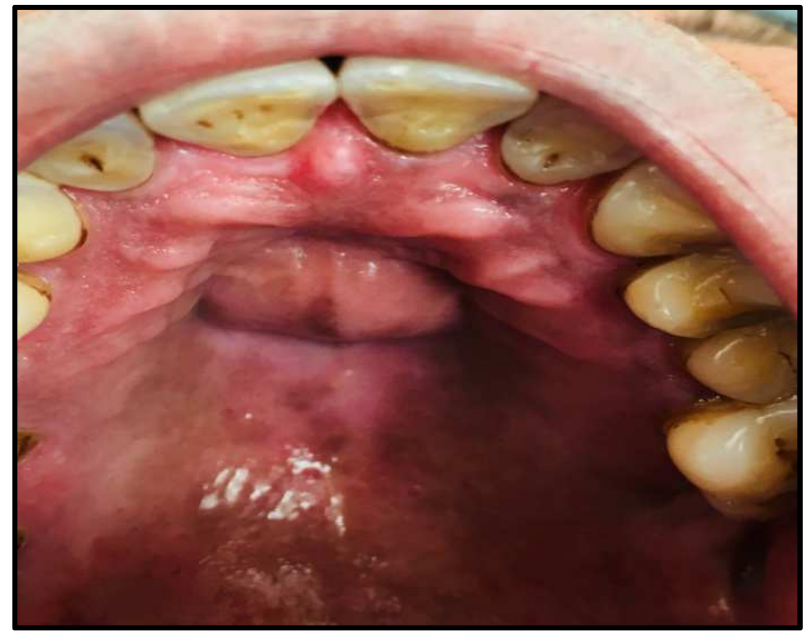

Figure-1: A solitary well-defined swelling in midpalatine region

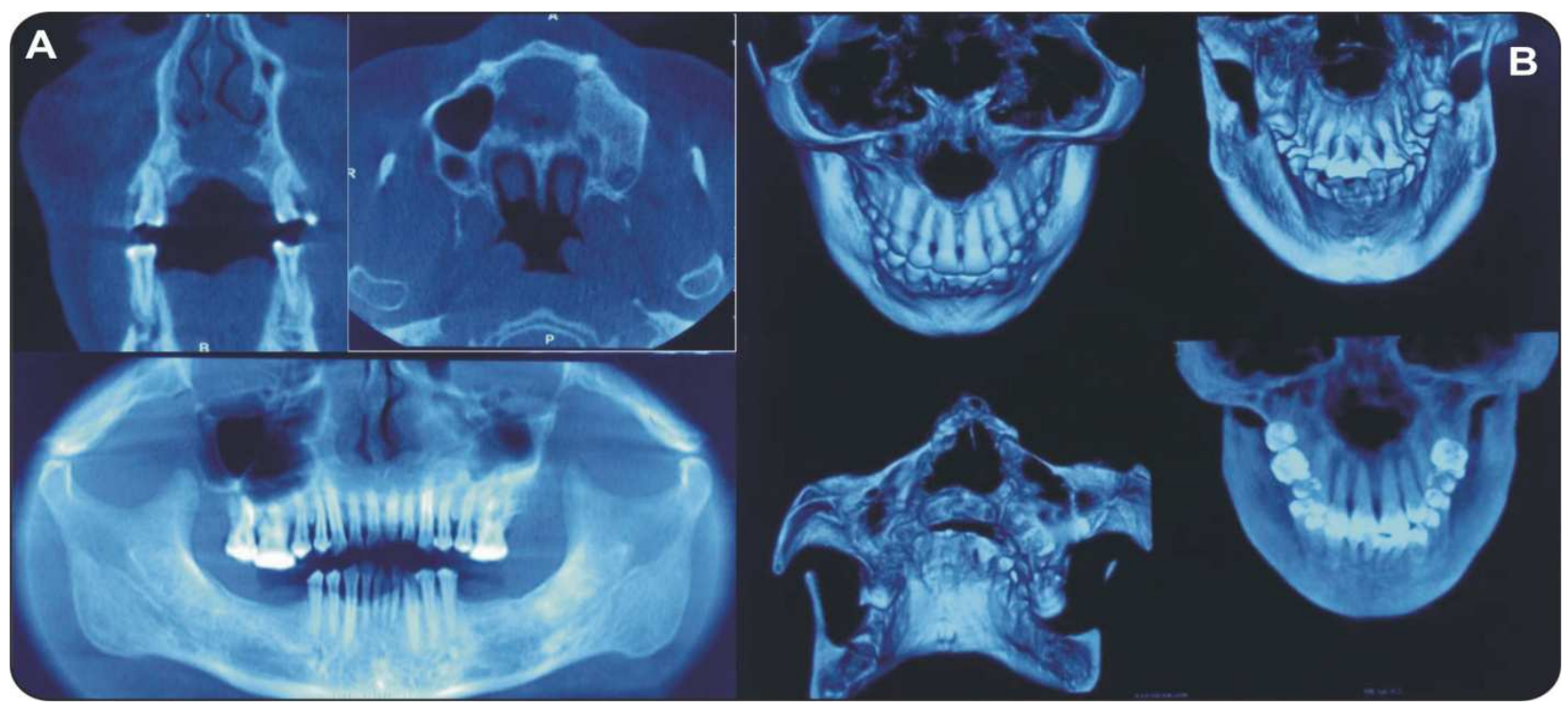

Figure-2: Multiplanar imaging and reformatting (A) Coronal section (B) Axial section $\mathbf{C}(\mathrm{C})$ 3-dimensional reformatted images showing well defined radiolucency in mid palatine region involving nasal floor and destructing labial cortical plates. 


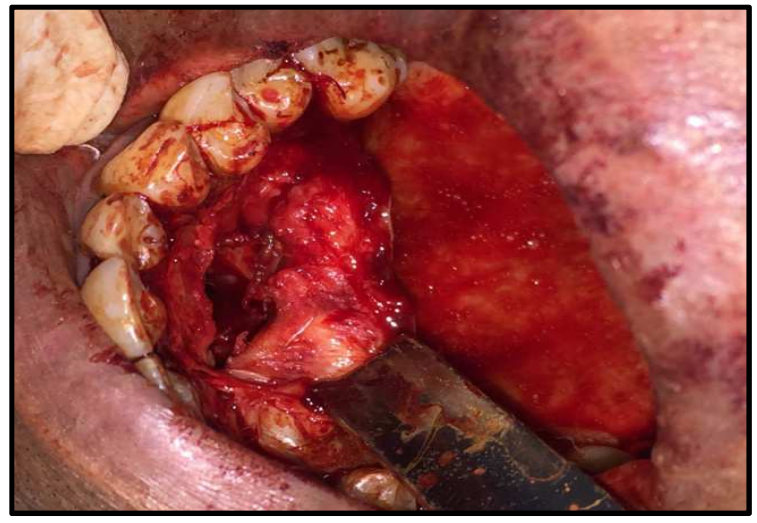

Figure-3: Cyst enucleation

\section{Case Report}

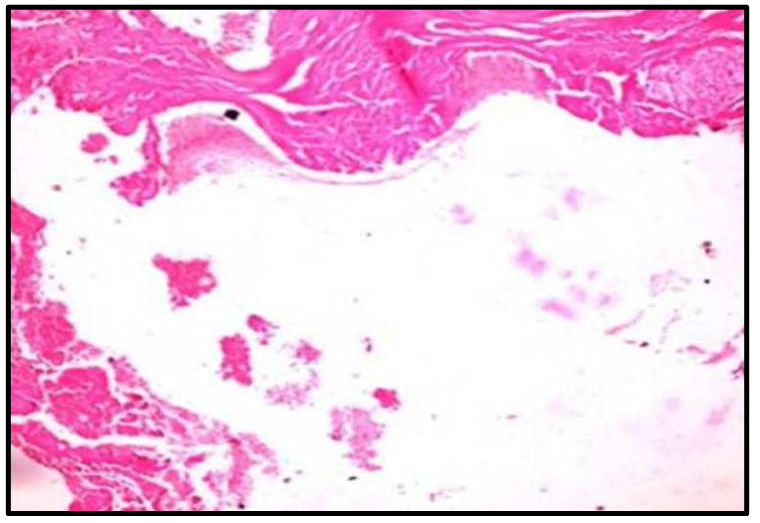

Figure-4: H \& E stained histopathologic section

On the basis of history, clinical and radiographic evidence, provisional diagnosis of NPDC was made, with differential diagnosis of median palatine cyst, periapical cyst and giant cell granuloma. All routine preliminary investigations were within normal range. Cyst enucleation was done under local anesthesia, crevicular incision was given and palatal mucoperiosteal flap was raised, complete cystic lining and contents were removed and repositioning of mucosal lining was performed (Figure 3). Interrupted sutures (3-0 silk) were placed and specimen was sent forhistopathological examination. The patient was prescribed routine antibiotics and analgesics and was recalled after 1 week for follow up and reported no complications.

The histopathological report revealed cystic epithelial lining composed of mainly stratified squamous epithelium with very few areas of simple cuboidal epithelium, with epithelial lining being 2-3 layers thick. The underlying connective tissue stroma was fibrous with bundles of interlacing collagen fibers, interspersed fibroblasts and chronic inflammatory cell infiltrate chiefly lymphocytes and plasma cells with few nerves and blood vessels in connective tissue, suggesting a final diagnosis of NPDC (Figure 4).

\section{Discussion}

The NPDC is one of the most common developmental, non-neoplastic, non-odontogenic cysts of the oral cavity, occurring in about $1 \%$ of population. They are believed to develop from remnants of paired embryonic nasopalatine ducts. NPDC is unique in that it develops in only a single location, which is the midline of the anterior maxilla. It can arise at any age, but is seen most often in patients between 30 and 60 years of age. It is slightly more common in males than in females, the ratio being $3: 1[1,2,5]$.

Nasopalatine ducts ordinarily undergoes progressive degeneration however, the persistence of the epithelial remnants may later become the source of epithelia that gives rise to NPDC, from either spontaneous proliferation or proliferation following trauma, bacterial infection or mucous retention. Genetic factors have also been suggested. The cyst is usually asymptomatic and discovered on routine radiographs. The most common presenting symptoms are swelling of the anterior palate, drainage and pain. Burning sensation and numbness may be experienced due to pressure on the nasopalatine nerve. Occasionally they cause intermittent discharge with a salty taste. Root resorption and displacement of teeth is a rare finding[5].
Radiographically, it can be detected on routine periapical and occlusal radiographs. It appears as a round or ovoid radiolucency between the roots of the central incisors. Due to superimposition of the nasal spine, a heart shaped appearance may be seen. Most of the lesions have a well defined sclerotic border. In some individuals, a prominent incisive canal can appear as a radiolucent area and mimic NPDC. Most authors agree that $6 \mathrm{~mm}$ should be considered the upper limit for normal incisive canal radiolucencies larger than this should be considered potentially pathologic and merit further investigation[3,4,7-9].

Multimodal tomography, can be used as an additional imaging modality as it exposes the patient to lesser radiation doses, employs crossed and sectional tomographic acquisitions in the sagittal plane to yield three dimensional images. Magnetic resonance imaging may also prove useful in establishing the diagnosis, and particularly contrast the interior of the NPDC with a high signal intensity[1,6].

A differential diagnosis must be established in order to avoid unnecessary treatments such as endodontic procedures in vital permanent upper central incisors. 


\section{Case Report}

List of differential diagnosis includes radicular cyst, lateral periodontal cyst, odontogenic cyst, central giant cell granuloma, ameloblastoma, odontogenicmyxoma and central hemangioma. A correct tentative diagnosis should be based on positive dental vitality testing and negative percussion findings of the permanent upper central incisors provided these teeth do not have pulp or periodontal problems[4].

Histopathological examination reveals a cavity lined by epithelium and surrounded by connective tissue wall. A reported $71.8 \%$ of NPDCs have squamous, columnar, cuboidal or some combination of these epithelial types; respiratory epithelium is seen in $9.8 \%$. The cyst wall may contain a chronic inflammatory reaction consisting of lymphocytes and plasma cells. Also helpful in the diagnosis of NPDC are the presence of neurovascular bundles, mucous glands and adipose tissue.Treatment of NPDC is surgical excision[4,10]. The present case showed typical clinical, radiological and histopathological features of NPDC.

\section{Conclusion}

Clinical and radiological presentation of NPDC can mimic common periapical lesion like radicular cyst, leading to its misdiagnosis. CBCT easily visualizes the radio-transparency on the midline, with well-defined sclerotic margins, and informs of the exact location of the lesion. In addition, it facilitates planning of the best surgical approach. Definitive diagnosis is established only after histological evaluation of the excised lining.

\section{Funding: Nil, Conflict of interest: None Permission of IRB: Yes}

\section{References}

1. Bezalwar A, Tiwari A, Maheshwari V, Patel N. Nasopalatine Duct Cyst: A Case Report. JDMS. 2015; 14 (6):60-3.
2. EscodaFrancolí J, Almendros Marqués N, Berini Aytés L, Gay Escoda C. Nasopalatine duct cyst: report of 22 cases and review of the literature. Med Oral Patol Oral Cir Bucal. 2008 Jul 1;13(7):E438-43.

3. Nilesh K, Vande AV, Suresh KV, Pramod RC, Suryavanshi P, Kadam NA. A Case of Nasopalatine Duct Cyst: Presentation, diagnosis and Management. J Dent App. 2016;3(2):325-27.

4. Shylaja S, Balaji K, Krishna A. Nasopalatine duct cyst: report of a case with review of literature. Indian J Otolaryngol Head Neck Surg. 2013 Dec;65(4):385-8. doi: 10.1007/s12070-011-0242-6. Epub 2011 Apr 11.

5. McCrea SJ. Nasopalatine Duct Cyst, a Delayed Complication to Successful Dental Implant Placement: Diagnosis and Surgical Management. JOI. 2014;XL (2): 189-94.

6. Pavankumar K, Sholapurkar AA, Joshi V. Surgical Management of Nasopalatine Duct Cyst: case report. Rev ClinPesqOdontol. 2010;6(1):81-6.

7. Allmendinger A, Gabe M, Destian S. Median palatine cyst. J Radiol Case Rep. 2009;3(7):7-10. doi: 10.3941/ jrcr. v3i7. 269. Epub 2009 Jul 1.

8. Salgado H, Felino A, Mesquita P. Extensive nasopalatine cyst with nasal involvement. RPEMD. 2014; 55 (3);171-76.

9. Yogesh TL, Shetty A, Nagaraj T, Sinha P. Nasopalatine duct cyst: A case report. JCRI. 2014; 1(2): 57-60.

10. Agarwal S, Jagade M, Thorawade V, Mishra A, Joshi S, Ahire D. Palatal Cyst: An Unusual Case Report. IJOHNS. 2013;2(1):39-41.

\section{How to cite this article?}

Pandya D, Dey S, Bhattacharya M, Singh P. Aggressive nasopalatine duct cyst with nasal involvement. Int J Med Res Rev 2018;6 (03):197-200. doi:10.17511/ijmrr. 2018.i03.11. 